\title{
Correction to: The dietary inflammatory index is associated with anti and pro-inflammatory adipokines in Brazilian schoolchildren
}

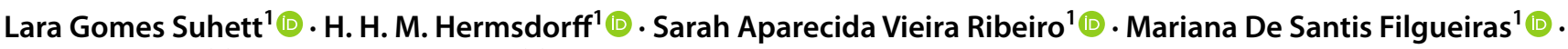 \\ Nitin Shivappa ${ }^{2,3,4}$. James R. Hébert ${ }^{2,3,4}$. J Juliana Farias de Novaes ${ }^{1}(1)$
}

Published online: 13 April 2021

(c) Springer-Verlag GmbH Germany, part of Springer Nature 2021

\section{Correction to: European Journal of Nutrition https://doi.org/10.1007/s00394-021-02500-8}

The original version of this article unfortunately contained a mistake. The presentation of Table 4 was incorrect and the legend of Table 1 is wrong in the article because those variables are not adjustment, and the P-value is from linear regression models, and not from Cochran-Armitage test. The corrected Tables 1 and 4 are given in the following page.

The original article can be found online at https://doi.org/10.1007/ s00394-021-02500-8.

Lara Gomes Suhett

nutrilarasuhett@gmail.com

1 Department of Nutrition and Health, Universidade Federal de Viçosa (UFV), Av. P.H. Rolfs s/n, Campus Universitário, Viçosa, Minas Gerais 36570-000, Brazil

2 Department of Epidemiology and Biostatistics, Arnold School of Public Health, University of South Carolina, Columbia, SC, USA

3 Statewide Cancer Prevention and Control Program (CPCP), Arnold School of Public Health, University of South Carolina, 915 Greene Street, Columbia, SC 29208, USA

4 Department of Nutrition, Connecting Health Innovations (CHI), 1417 Gregg Street, Columbia, SC 29201, USA 
Table 1 Distribution of Children's Dietary Inflammatory Index (C-DII) according to sociodemographic and behavior characteristics in schoolchildren from Viçosa,

Minas Gerais, Brazil, 2015

\begin{tabular}{|c|c|c|c|c|}
\hline \multirow[t]{2}{*}{ Characteristics } & \multirow[t]{2}{*}{$n$} & \multicolumn{2}{|l|}{ C-DII } & \multirow[t]{2}{*}{$P$ value $^{\mathrm{b}}$} \\
\hline & & Mean \pm SD & Mean difference (95\% CI) & \\
\hline \multicolumn{5}{|l|}{ Sex } \\
\hline Male & 181 & $0.6 \pm 0.9$ & Reference & \multirow[t]{2}{*}{0.47} \\
\hline Female & 197 & $0.5 \pm 0.9$ & $-0.1(-0.3,0.1)$ & \\
\hline \multicolumn{5}{|l|}{ Age (years) } \\
\hline 8 & 183 & $0.5 \pm 0.9$ & Reference & \multirow[t]{2}{*}{0.58} \\
\hline 9 & 195 & $0.6 \pm 0.9$ & $0.1(-0.1,0.2)$ & \\
\hline \multicolumn{5}{|l|}{ Ethnicity } \\
\hline White & 119 & $0.3 \pm 0.9$ & Reference & \multirow[t]{2}{*}{$0.001^{*}$} \\
\hline Non-white & 259 & $0.7 \pm 0.9$ & $0.3(0.1,0.5)$ & \\
\hline \multicolumn{5}{|l|}{ School } \\
\hline Private & 110 & $0.3 \pm 1.0$ & Reference & \multirow[t]{2}{*}{$<0.001 *$} \\
\hline Public & 268 & $0.7 \pm 0.9$ & $0.3(0.1,0.5)$ & \\
\hline \multicolumn{5}{|l|}{ Per capita income (US\$) ${ }^{\mathrm{a}}$} \\
\hline$<96.9$ (1st quartile) & 93 & $0.7 \pm 0.9$ & Reference & \multirow[t]{4}{*}{$0.001 *$} \\
\hline $97.0<155.3$ (2nd quartile) & 94 & $0.7 \pm 0.9$ & $0.0(-0.3,0.2)$ & \\
\hline $155.4<262.2$ (3rd quartile) & 97 & $0.6 \pm 0.9$ & $-0.1(-0.3,0.2)$ & \\
\hline$>262.3$ (4th quartile) & 94 & $0.3 \pm 1.0$ & $-0.4(-0.6,-0.1)$ & \\
\hline \multicolumn{5}{|l|}{ Screen time (hours/day) } \\
\hline$\leq 2$ & 198 & $0.5 \pm 0.9$ & Reference & \multirow[t]{2}{*}{0.22} \\
\hline$>2$ (sedentary behavior) & 180 & $0.6 \pm 1.0$ & $0.1(-0.1,0.3)$ & \\
\hline
\end{tabular}

SD standard deviation, $95 \%$ CI 95\% confidence interval

$* P<0.05$

${ }^{a}$ Approximate exchange rates of real (R\$) to dollar (US\$) at the time of this study (US\$1.00 $=\mathrm{R} \$ 3.22$ )

${ }^{b}$ From linear regression models. For variables with three or more categories, $P$ value was from Wald test for linear trend representing ordinal categories as continuous 
Table 4 Crude and adjusted analyses of the association between Children's Dietary Inflammatory Index (C-DII) and adipokines in schoolchildren from Viçosa, Minas Gerais, Brazil, 2015

\begin{tabular}{|c|c|c|c|c|c|c|c|}
\hline \multirow[t]{2}{*}{ Adipokines } & \multicolumn{5}{|c|}{ C-DII (quintiles) } & \multirow[t]{2}{*}{ Per 1 SD } & \multirow[t]{2}{*}{$P$ trend $^{\mathrm{d}}$} \\
\hline & $\begin{array}{l}\mathrm{Q} 1(-2.16 \\
\text { to }-0.19) \\
(n=75)\end{array}$ & $\begin{array}{l}\mathrm{Q} 2(-0.20 \text { to } \\
0.41)(n=76)\end{array}$ & $\begin{array}{l}\mathrm{Q} 3(0.42-0.86) \\
(n=76)\end{array}$ & $\begin{array}{l}\text { Q4 (0.87-1.41) } \\
(n=76)\end{array}$ & $\begin{array}{l}\text { Q5 (1.42-2.75) } \\
(n=75)\end{array}$ & & \\
\hline \multicolumn{8}{|c|}{ Adiponectin $(\mu \mathrm{g} / \mathrm{mL})$} \\
\hline Mean \pm SD & $14.5 \pm 7.7$ & $14.6 \pm 7.8$ & $12.4 \pm 6.3$ & $13.6 \pm 7.5$ & $12.6 \pm 7.5$ & & \\
\hline $\begin{array}{l}\text { Unadjusted dif- } \\
\text { ference ( } 95 \% \\
\text { CI })^{\mathrm{a}}\end{array}$ & Reference & $0.2(-2.3,2.6)$ & $-2.0(-4.3,0.2)$ & $-0.8(-3.3,1.6)$ & $-1.8(-4.3,0.6)$ & $\begin{array}{l}-0.7(-1.5,- \\
0.04)\end{array}$ & $0.04 *$ \\
\hline $\begin{array}{l}\text { Adjusted dif- } \\
\text { ference }(95 \% \\
\text { CI) }\end{array}$ & Reference & $0.1(-2.4,2.6)$ & $-2.2(-4.5,0.1)$ & $-1.0(-3.5,1.5)$ & $-1.9(-4.4,0.6)$ & $\begin{array}{l}-0.8(-1.6,- \\
0.1)\end{array}$ & $0.04 *$ \\
\hline $\begin{array}{l}\text { Adjusted dif- } \\
\text { ference }(95 \% \\
\text { CI) }\end{array}$ & Reference & $0.2(-2.2,2.7)$ & $-2.0(-4.3,0.2)$ & $-0.8(-3.2,1.7)$ & $-1.9(-4.4,0.6)$ & $\begin{array}{l}-0.8(-1.5,- \\
0.03)\end{array}$ & $0.04^{*}$ \\
\hline \multicolumn{8}{|l|}{ Leptin (ng/mL) } \\
\hline Mean \pm SD & $6.0 \pm 11.3$ & $5.7 \pm 8.9$ & $5.0 \pm 7.7$ & $6.8 \pm 10.6$ & $5.0 \pm 8.5$ & & \\
\hline $\begin{array}{l}\text { Unadjusted dif- } \\
\text { ference ( } 95 \% \\
\text { CI) }\end{array}$ & Reference & $-0.3(-3.6,2.9)$ & $-1.0(-4.1,2.0)$ & $0.8(-2.7,4.3)$ & $1.0(4.2,2.2)$ & $0.0(-0.9,0.9)$ & 0.91 \\
\hline $\begin{array}{l}\text { Adjusted dif- } \\
\text { ference }(95 \% \\
\text { CI })^{\mathrm{b}}\end{array}$ & Reference & $-0.3(3.6,3.0)$ & $-0.8(-4.1,2.5)$ & $1.0(-2.6,4.6)$ & $0.9(-4.3,2.5)$ & $0.0(-0.9,1.1)$ & 0.90 \\
\hline $\begin{array}{l}\text { Adjusted dif- } \\
\text { ference }(95 \% \\
\text { CI) }\end{array}$ & Reference & $-1.3(-4.1,1.4)$ & $-1.7(-4.3,1.0)$ & $-0.5(-3.4,2.4)$ & $-0.7(-3.3,1.9)$ & $-0.1(-0.8,0.6)$ & 0.90 \\
\hline \multicolumn{8}{|l|}{$\mathrm{RBP} 4(\mu \mathrm{g} / \mathrm{mL})$} \\
\hline Mean \pm SD & $4.2 \pm 0.9$ & $4.2 \pm 0.7$ & $3.9 \pm 0.5$ & $4.0 \pm 0.6$ & $3.8 \pm 0.5$ & & \\
\hline $\begin{array}{l}\text { Unadjusted dif- } \\
\text { ference (95\% } \\
\text { CI) })^{\mathrm{a}}\end{array}$ & Reference & $0.0(-0.3,0.2)$ & $\begin{array}{l}-0.3(-0.6,- \\
0.1)\end{array}$ & $\begin{array}{l}-0.2(-0.5,- \\
0.1)\end{array}$ & $\begin{array}{l}-0.4(-0.7,- \\
0.2)\end{array}$ & $\begin{array}{l}-0.1(-0.2,- \\
0.1)\end{array}$ & $<0.001 *$ \\
\hline $\begin{array}{l}\text { Adjusted dif- } \\
\text { ference }(95 \% \\
\text { CI })^{\mathrm{b}}\end{array}$ & Reference & $0.0(-0.3,0.3)$ & $-0.3(-0.6,0.0)$ & $-0.2(-0.5,0.0)$ & $\begin{array}{l}-0.4(-0.7,- \\
0.2)\end{array}$ & $\begin{array}{l}-0.1(-0.2,- \\
0.05)\end{array}$ & $0.001 *$ \\
\hline $\begin{array}{l}\text { Adjusted dif- } \\
\text { ference }(95 \% \\
\text { CI })^{\mathrm{c}}\end{array}$ & Reference & $0.0(-0.3,0.2)$ & $\begin{array}{l}-0.3(-0.6,- \\
0.1)\end{array}$ & $-0.2(-0.5,0.0)$ & $\begin{array}{l}-0.4(-0.7,- \\
0.2)\end{array}$ & $\begin{array}{l}-0.1(-0.2,- \\
0.05)\end{array}$ & $0.001 *$ \\
\hline \multicolumn{8}{|l|}{ Chemerin (ng/mL) } \\
\hline Mean \pm SD & $75.0 \pm 65.4$ & $74.6 \pm 58.2$ & $89.7 \pm 68.9$ & $89.3 \pm 71.1$ & $89.2 \pm 58.9$ & & \\
\hline $\begin{array}{l}\text { Unadjusted dif- } \\
\text { ference (95\% } \\
\text { CI) }\end{array}$ & Reference & $\begin{array}{l}-0.4(-20.3 \\
19.4)\end{array}$ & $14.6(-6.8,36.1)$ & $14.3(-7.5,36.1)$ & $14.2(-5.7,34.2)$ & $6.1(-0.5,12.7)$ & 0.07 \\
\hline $\begin{array}{l}\text { Adjusted dif- } \\
\text { ference }(95 \% \\
\text { CI })^{\mathrm{b}}\end{array}$ & Reference & $1.1(-19.1,21.2)$ & $18.4(-3.1,39.9)$ & $17.3(-5.2,39.7)$ & $16.6(-4.6,37.8)$ & $7.4(0.4,14.4)$ & $0.04 *$ \\
\hline $\begin{array}{l}\text { Adjusted dif- } \\
\text { ference }(95 \% \\
\text { CI) }\end{array}$ & Reference & $\begin{array}{l}-0.1(-20.4 \\
20.2)\end{array}$ & $17.3(-4.1,38.8)$ & $16.3(-6.5,38.1)$ & $17.6(-4.2,37.8)$ & $7.2(0.3,14.1)$ & $0.04^{*}$ \\
\hline
\end{tabular}

95\% CI 95\% confidence interval, $Q$ quintiles, $S D$ standard deviation

$P<0.05^{*}$

${ }^{a}$ From linear regression models with each adipokine as a continuous outcome and C-DII as predictor. Robust estimates of variance were specified in all models

${ }^{b}$ From linear regression adjusted for child's age, sex, race, per capita income and screen time

${ }^{c}$ From linear regression adjusted for child's age, sex, race, per capita income, screen time and $\%$ body fat

${ }^{\mathrm{d}}$ For a variable representing ordinal C-DII categories introduced as a continuous predictor 